\title{
Association of Joubert Syndrome and Hirschsprung Disease
}

\author{
Radheshyam Purkait, Rajarshi Basu, Rituparna Das ANd *Uttara ChatterJee \\ From Department of Paediatric Medicine, NRS Medical College and Hospital, Kolkata; and *Department of Pathology, IPGMER \\ and SSKM Hospital, Kolkata, India.
}

Correspondence to: Dr Radheshyam Purkait, Associate Professor, Department of Paediatric Medicine, NRS Medical College \& Hospital, Kolkata 700 014, West Bengal, India.radheshyampurkait@gmail.com Received: August 08, 2014; Initial review: September 08, 2014; Accepted: October 09, 2014.
Background: Association between Joubert Syndrome and Hirschsprung disease is rare. Case characteristics: A 9-month-old girl having developmental delay and chronic constipation. Observation: Molar tooth sign on MRI brain and absence of ganglion cells in rectal biopsy specimen. Outcome: Child underwent surgical repair for Hirschsprung disease. Message: Association of these two rare entities could be explained by ciliopathy.

Keywords: Ciliopathy, Constipation, Developmental delay.
$\mathrm{J}$ oubert syndrome is an autosomal recessive or $\mathrm{X}$ linked congenital abnormality of cerebellar vermis and brain stem that is clinically recognized in infancy [1,2]. Hirschsprung disease is congenital malformation of the hindgut that manifests clinically as intestinal obstruction [3]. Association between Joubert syndrome and Hirschsprung disease is a rare phenomenon [4]. Here we report a 9-month-old female infant who had typical clinical and radiological findings of these two diseases.

\section{Case Report}

A 9-month-old girl was brought with the complaints of delay in the developmental milestones, abnormal eye movements and chronic constipation. Her parents had also noticed abnormal breathing pattern during her early neonatal period. There was no history of seizures and feeding or swallowing difficulty. She was born at term out of consanguineous marriage with an uneventful perinatal period. Past history revealed a stormy neonatal period with admission to neonatal intensive care unit for vomiting, abdominal distension and delayed passage of meconium. Child was diagnosed to have late-onset sepsis and was discharged home with the advice to attend followup clinic at regular interval. She had no other siblings. There was no history of neurologic, genetic or gastrointestinal problems in other family members.

Examination revealed facial dysmorphism in the form of prominent forehead, high rounded eyebrows, low set ears, depressed nasal bridge, hypertelorism, and right sided ptosis, nystagmus and lateral squint. Abdomen was distended but there was no organomegaly. Neurological examination showed a hypotonic child with preserved tendon reflexes. Beside right-sided ptosis and lateral squint, examination of the cranial nerves was normal. Rest of the systemic examination was normal.

Complete blood count and routine biochemical tests were within normal ranges. Thyroid profile was noncontributory. Magnetic resonance imaging (MRI) of brain revealed disorganized cerebellar vermis with thickened superior cerebellar peduncles around the 4th ventricle forming the classical molar tooth sign (Fig. 1). A conventional $\mathrm{G}$ banding chromosomal study showed a normal karyotype (46, XX). As an initial work-up of chronic constipation, both $X$-ray abdomen and ultrasonography of abdomen was done which showed distended transverse colon, especially left lateral third along with distended splenic flexure. Barium enema revealed distended rectum, sigmoid colon and proximal transverse colon. Significant amount of barium was present in the colon on twenty-four hour delayed films. Rectal biopsy showed a large number of hypertrophied nerve bundles with absence of ganglion cells consistent with the diagnosis of Hirschsprung disease (Fig. 2). Subsequently child underwent definite surgical repair for Hirschsprung disease with uneventful post-operative period.

\section{Discussion}

The neuroradiological hallmark of Joubert syndrome is the characteristic molar tooth sign, a peculiar malformation of the midbrain-hindbrain junction, visible on axial MRI films. Joubert syndrome and related disorders is classified into six phenotypic subgroups: pure, with ocular defect, with renal defect, with oculorenal defects, with hepatic defect, and with orofaciodigital defects [2]. The pathogenetic mechanism of Joubert syndrome includes mutation in genes encoding 


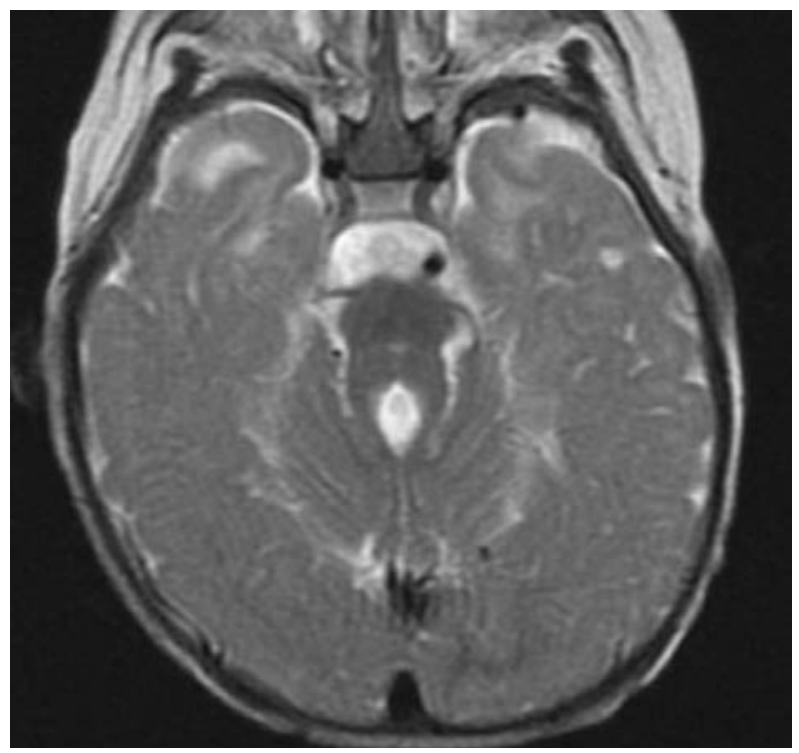

FIG. 1 T2W MRI showing hypoplasia of the cerebellar vermis with elongated and thickened superior cerebellar peduncles producing “molar tooth sign”.

proteins of the primary cilium or its apparatus. The term "ciliopathy" usually refers to a group of genetic disorders affecting the cellular cilia or the ciliary anchors, namely the basal bodies resulting in improper ciliary function through proteolytic cleavage of ciliary proteins. Proper functioning of primary cilia is known to be instrumental in the development and functioning of several cell types, including retinal photoreceptors, neurons, kidney tubules and bile ducts $[5,6]$. These organelles have been implicated both in neuronal cell proliferation and axonal migration [7].

We diagnosed Hirschsprung disease in our patient with characteristic clinical, radiological and histo-pathological findings. It is considered to be the result of premature arrest of the craniocaudal migration of vagal neural cest cells in the hindgut between the 5th and 12th week of gestation to form the enteric nervous system, and is therefore regarded as a neurocristopathy [8]. Hirschsprung disease has also been found to be associated with another ciliopathy, Bardet-Biedl syndrome [9]. As cilia are involved in the development of neural crest [10], ciliopathy may be the common underlying pathogenic mechanism accountable for both the central nervous system anomalies in Joubert syndrome and neurocristopathy in Hirschsprung disease.

Contributors: RP: diagnosed, worked up the case and wrote the manuscript; RB, RD: managed and followed up the case; RP, UC: reviewed the literature and prepared the final manuscript. Funding: None; Competing interests: None stated.

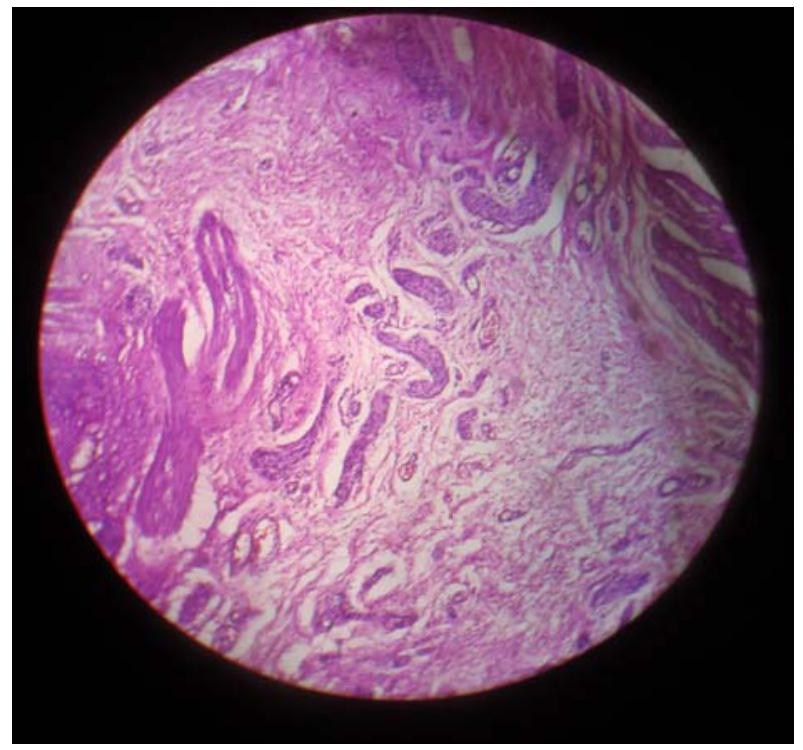

FIG. 2 Rectal suction biopsy showing a large number of hypertrophied nerve bundles with an absence of ganglion cells suggestive of HD [H and E stain. X10]. (See website for color image).

\section{REFERENCES}

1. Romani M, Micalizzi A, Valente EM. Joubert syndrome: Congenital cerebellar ataxia with the molar tooth. Lancet Neurol. 2013;12:894-905.

2. Brancati F, Dallapiccola B, Valente EM. Joubert Syndrome and related disorders. Orphanet J Rare Dis. 2010;5:20.

3. Amiel J, Sproat-Emison E, Garcia-Barcelo M, Lantieri F, Burzynski G, Borrego S, et al. Hirschsprung disease, associated syndromes and genetics: A review. J Med Genet. 2008;45:1-14.

4. Ozyurek H, Kayacik OE, Gungor O, Karagoz F. Rare association of Hirschsprung's disease and Joubert syndrome. Eur J Pediatr. 2008;167:475-7.

5. Lancaster MA, Gleeson JG. The primary cilium as a cellular signaling center: Lessons from disease. Curr Opin Genet Dev. 2009;19:220-9.

6. Badano JL, Mitsuma N, Beales PL, Katsanis N. The ciliopathies: An emerging class of human genetic disorders. Ann Rev Genomics Hum Genet. 2006;7:125-48.

7. Millen KJ, Gleeson JG. Cerebellar development and disease. Curr Opin Neurobiol. 2008;18:12-9.

8. Taraviras S, Pachnis V. Development of the mammalian enteric nervous system. Curr Opin Genet Dev. 1999;9:3217.

9. Lorda-Sanchez I, Ayuso C, Ibañez A. Situs inversus and Hirschsprung disease: Two uncommon manifestations in Bardet-Biedl syndrome. Am J Med Genet. 2000;90:80-1.

10. de Pontual L, Zaghloul NA, Thomas S, Davis EE, McGaughey DM, Dollfus H, et al. Epistasis between RET and BBS mutations modulates enteric innervation and causes syndromic Hirschsprung disease. Proc Natl Acad Sci USA. 2009;106:13921-6. 